\title{
Sex Specific Effects of Prunella vulgaris on Longevity Regulation
}

\author{
Jie Shen ${ }^{1}$ (D) Boying Liang ${ }^{1}$ \\ Accepted: 29 December 2021 / Published online: 8 January 2022 \\ (c) The Author(s), under exclusive licence to Springer Science+Business Media, LLC, part of Springer Nature 2022
}

\begin{abstract}
The sudden outbreak of COVID-19 in over 200 countries globally, brought serious effects on our daily lives. Traditional Chinese Medicine (TCM) in combination with regional characteristics has achieved remarkable results in China. Prunella vulgaris is one of the ingredients of commercially available canned Chinese herbal tea which demonstrated lifespan extending effect on Drosophila in our previous study, whereas it is unknown which ingredients contribute to it. This study used Drosophila as the model to explore whether Prunella vulgaris is the component taking effect on Drosophila longevity. Results showed that Prunella vulgaris can extend the lifespan of female flies by $10.42 \%$, and improve their endurance under heat stress by $18.46 \%$.
\end{abstract}

Keywords Prunella vulgaris $\cdot$ Traditional Chinese Medicine $\cdot$ Sex specificity

\section{Introduction}

TCM uses a combination of multiple herbal medicines for disease prevention and treatment, which is changeable and complicated. We previously found the commercially available canned Chinese herbal tea, a kind of medicinal tea, can increase longevity of Drosophila [1]. But it is unknown which ingredients contribute to it. Prunella vulgaris is one of the ingredients of the Chinese herbal tea, and has been used for the treatment of hepatitis, tuberculosis and diabetes through the ages [2]. Based on the literature, we found that Prunella vulgaris might be the ingredient have the potential beneficial effect.

\section{Materials and Methods}

We used Drosophila melanogaster wild type Canton-S for the experiments. Collected eclosing adult flies over an eighthour period. Flies were mated for $48 \mathrm{~h}$ before sorting into male vials or female vials (40 flies per vial, $n=3$ ) on day 0 of the life span assay. The flies were maintained on a 12-h light/dark cycle in an incubator at $25^{\circ} \mathrm{C}$. To prepare the

Jie Shen

shenjie@hdu.edu.cn

1 College of Artificial Intelligence, Hangzhou Dianzi

University, Hangzhou 310018, China solution of Prunella vulgaris, adding $1.5 \mathrm{~g}$ Prunella vulgaris to $60 \mathrm{~mL}$ water in a $100 \mathrm{~mL}$ reaction caldron at $100{ }^{\circ} \mathrm{C}$ for $3 \mathrm{~h}$ without stirring, using $22 \mu \mathrm{m}$ filter to get the original supernatant for testing. Then three concentrations including original supernatant, 1:10 dilution and 1:100 dilution were tested. Flies were fed on Prunella vulgaris of original supernatant for 10 days used for the assay below.

\section{Results and Discussion}

The effect of Prunella vulgaris on regulation of lifespan of Drosophila is shown in Fig. 1. When the highest concentration of Prunella vulgaris was supplied in the food medium, the average lifespan of female flies was increased by $10.54 \%$, $p<0.05$ (Fig. 1A); but it did not increase lifespan in males (Fig. 1B). As an ingredient of herbal tea, it is reasonable that the percentage of increase is not big. For the result of heat stress assay, prunella vulgaris with the highest concentration can prolong the female lifespan by $18.46 \%(p<0.05)$ under thermal stress (Supplementary Fig. 1), indicating that Prunella vulgaris reduced stress damage caused by heat stress. The results showed that Prunella vulgaris can prolong the lifespan of flies. But it is sex-specific. Prunella vulgaris did not significantly affect the food intake and 24-h activity (Supplementary Fig. 2 and Supplementary Table 1). Phytochemical studies show that Prunella vulgaris is rich 
Fig. 1 Effect of Prunella vulgaris on regulation of survival in females (A) and males (B)

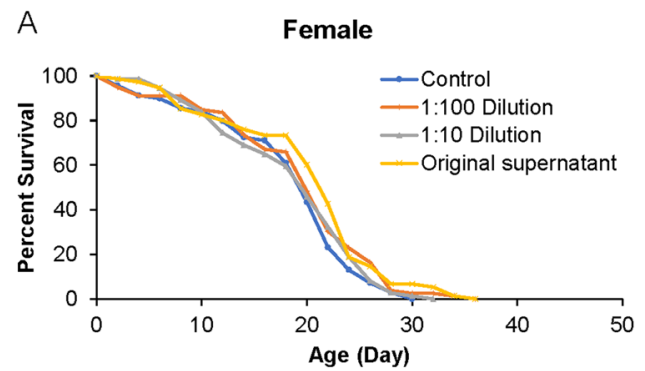

B Male

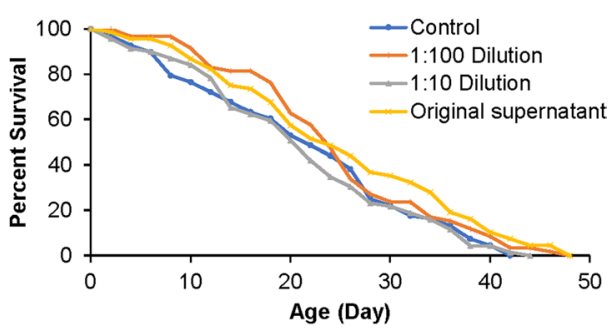

in bioactive chemicals, including triterpenoids, flavonoids, phenolic acids, organic acids, sterols, and sugars [3].

Prunella vulgaris can induce the activation of nuclear factor E2-related factor 2 (Nrf2) [4]. There are also research showing that Prunella vulgaris extracts can enhances Hsp70 expression [5]. As heat shock proteins are prominent members of vitagene network in neuroprotection [6], and in this research we observed enhanced stress resistance, the possible mechanism of Prunella vulgaris on lifespan regulation could be through hormesis, the activation of the Nrf2/ARE pathway and up upregulation of vitagenes, including heat shock proteins. Hormetic response by phytochemicals may increase cellular protection against challenges, and promote longevity. Sex specificity was also observed in our results and it would be interesting to know how it happened. Future research to clarify the mechanisms of lifespan extension of Prunella vulgaris would make sense.

Supplementary Information The online version contains supplementary material available at https://doi.org/10.1007/s11130-022-00950-x.

Acknowledgements This work was supported by the Grant from National Natural Science Foundation of China (31500970 to J.S.).

Data Availability Data is available upon request.

\section{Declarations}

Conflicts of Interest The authors declare that they have no conflict of interest.

\section{References}

1. Shen J, Shan J, Zhu X, Xu Y, Li M (2020) Effect of chinese herbal tea on health tested on Drosophila model. Plant Foods Hum Nutr 75(2):305-306

2. Chen Y, Zhang X, Guo Q et al (2019) Plant morphology, physiological characteristics, accumulation of secondary metabolites and antioxidant activities of Prunella vulgaris $\mathrm{L}$. under UV solar exclusion. Biol Res 52(1):17. https://doi.org/10.1186/ s40659-019-0225-8

3. Ryu SY, Oak MH, Yoon SK et al (2000) Anti-allergic and antiinflammatory triterpenes from the herb of Prunella vulgaris.[J]. Planta Med 66(4):358-360

4. Hwang SM, Lee YJ, Yoon JJ, Lee SM, Kim JS, Kang DG et al (2012) Prunella vulgaris suppresses HG-induced vascular inflammation via Nrf2/HO-1/eNOS activation. Int J Mol Sci 13(1):1258-1268

5. Pan W, Li Z, Lun F et al (2014) Effects of extracts of Prunella vulgaris $\mathrm{L}$. on proteome of human lung adenocarcinoma cell line A549. [J] Zhonghua Yi Xue Za Zhi 94(28):2216-2221

6. Dattilo S, Mancuso C, Koverech G, Di Mauro P, Ontario ML, Petralia CC et al (2015) Heat shock proteins and hormesis in the diagnosis and treatment of neurodegenerative diseases. Immun Ageing 12:20

Publisher's Note Springer Nature remains neutral with regard to jurisdictional claims in published maps and institutional affiliations. 